\title{
ANATYSIS AND MEASUREMENT OF CROSS-TALK IN A SUPERCONDUCTING CAVITY
}

\author{
Yongxiang Zhao \\ Brookhaven National Laboratory, Upton, New York
}

\begin{abstract}
Work performed under the auspices of the U.S. Department of Energy under Contract No. DE-AC02-98CH10886.
\end{abstract}

September 2002 


\title{
ANALYSIS AND MEASUREMENT OF CROSS-TALK IN A SUPERCONDUCTING CAVITY
}

\author{
Yongxiang Zhao \\ Physics Department, Bldg. 901-A \\ Brookhaven National Laboratory, Upton, NY 11973
}

\begin{abstract}
A superconducting cavity used in a microwave gun requires that the launcher and the pickup probes be on the same side of the cavity, which causes direct coupling between them, or crosstalk. At room temperature, the crosstalk causes serious distortion of the RF response. This note addresses the phenomenon, the simulation results and the analysis, so that one can extract the desired information from the confusing signal.
\end{abstract}

\section{INTRODUCTION}

In the "Electron Cooling" project, a microwave gun is employed as an electron source, and a superconducting cavity (SCC) is used in the first experiment. Microwave guns have been successful in many places, such as at the ATF at BNL. Superconducting cavities also have been successfully employed in many laboratories around the world. However, to our knowledge, there is little experience of a SCC used in a microwave gun.

A SCC in an accelerator usually has its input antenna (or launcher) on one side and the pickup probe on the other side. The coupling between them is only through the cavity cells, and has a perceivable coupling only near the resonant frequency. For a cavity of a microwave gun, one end must be the cathode; thus, both launcher and pickup must be on the same side.

A schematic of the SCC is shown in Fig.1. Probe A is the input or launcher; probe B is the pickup; and probe $\mathrm{C}$ is an antenna used for temporary measurement only. For convenience of machining and welding, the launcher and pickup are diametrically opposite. The direct coupling generating a crosstalk becomes inevitable. It becomes more significant because of the fact that for a SCC the coupling between launcher and the cavity must be very weak at room temperature, and the external $Q$ must be very large in order to match the cavity when it is cooled down to superconducting status. When the cavity is superconducting, crosstalk may be negligible small, but we have to test all the parameters of the cavity at room temperature, where the crosstalk is troublesome.

Our first measurements found that the pickup signal had too much distortion from a resonant curve. Fig. 2 shows some typical response of S21. We determined that the abnormal phenomenon was due to crosstalk. To extract the useful information from the undesirable signal is a challenge.

To this end, we had to make a model for simulation. Besides, analyses are also necessary in order to understand the relationship between parameters and the responses in the measurements. The formulae for calculation are collected. Finally, test results of the mentioned cavity are presented. 


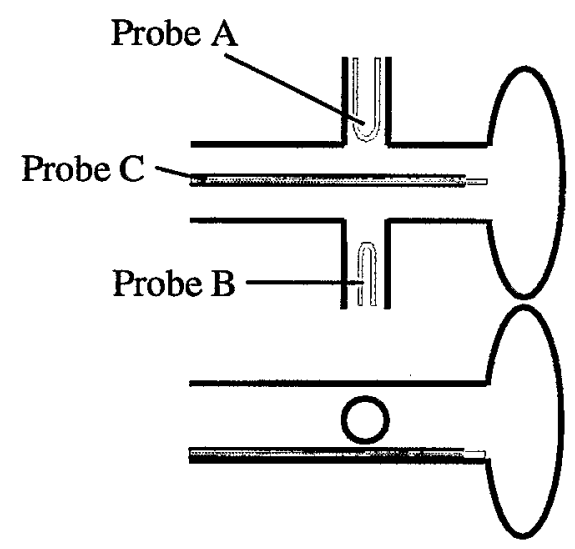

Fig. 1 Test setup

\section{PHENOMENA IN THE MEASUREMENT}

For a typical cavity with one port, the coupling and the $\mathrm{Q}$ value can be measured by the VSWR on the input line (or waveguide). However, the coupling in our case was too weak, so that the great majority of power reflects from the cavity and the conventional method results in too much error.

In the beginning, we measured the $S 21$ of the cavity by means of the network analyzer HP8753D, with ports 1 and 2 connected to the launcher and pickup (probes A and B in Fig.1), respectively. Fig. 2(a) shows the magnitude of S21 (in dB). The peak frequency is about $1.3 \mathrm{GHz}$ as expected, but the shape is far from the expected resonant curve. There is a dip frequency a little lower than the peak frequency. Near the peak the curve is markedly asymmetric so much so that the $Q$ value calculated by the network analyzer does not make sense. Fig. 2(b) shows its phase response. Instead of an "S" curve with 180 degree span as it should be, it has much less span and looks like a "stroke curve", making things more confusing.

The pickup probe was a loop, which usually is regarded as a magnetic coupler. In theory it should not couple with the launcher, which is an electric coupler. Unfortunately, this was not true. When we rotated the loop, the responses of S21 were different in all four quadrants. At one position, the dip frequency moved to the upper side, as shown in Fig. 2(c); meanwhile, the phase response was also reversed, as shown in Fig. 2(d).

The fact that the pickup signal depended on the rotation angle of the loop implies that the loop involves both magnetic and electric couplings, just like a loop directional coupler. This is understandable, because the loop is located in a region of electric field where the magnetic field is very weak.

We found the crosstalk in this structure to be inevitable. Fortunately, we recognize that this crosstalk will diminish when the cavity is cooled down to its superconducting state (See later for detail.). We then replace the loop pickup by another probe for easier machining and control. 


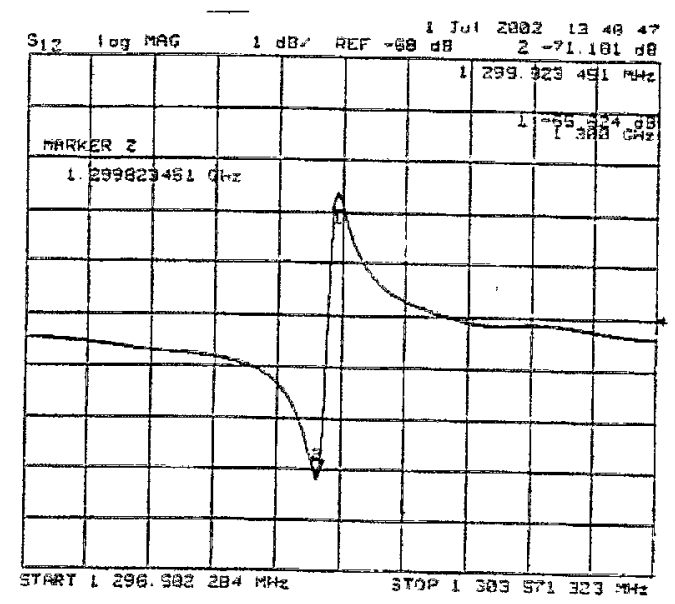

(a)

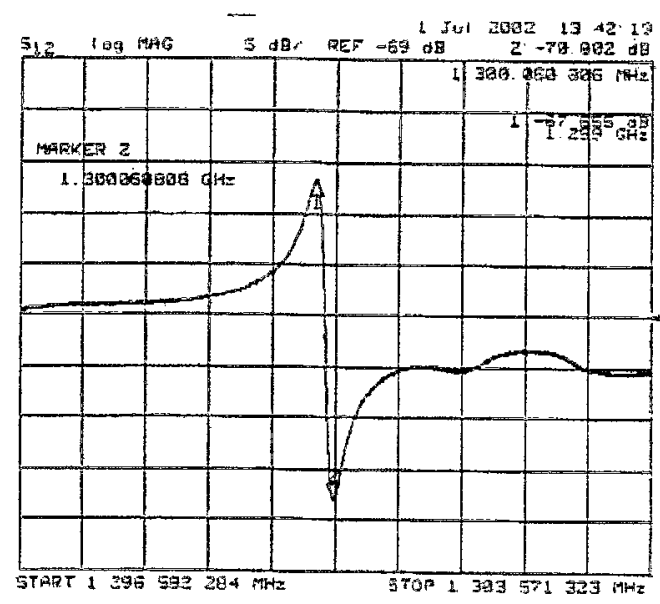

(c)

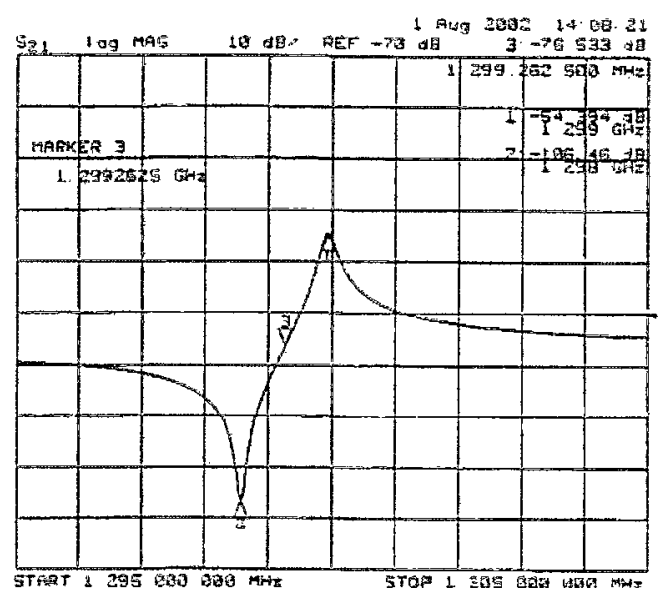

(e)

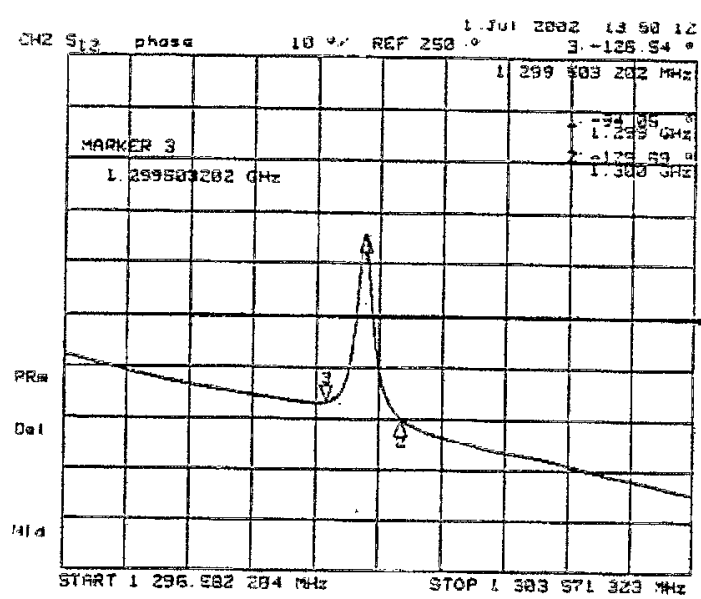

(b)

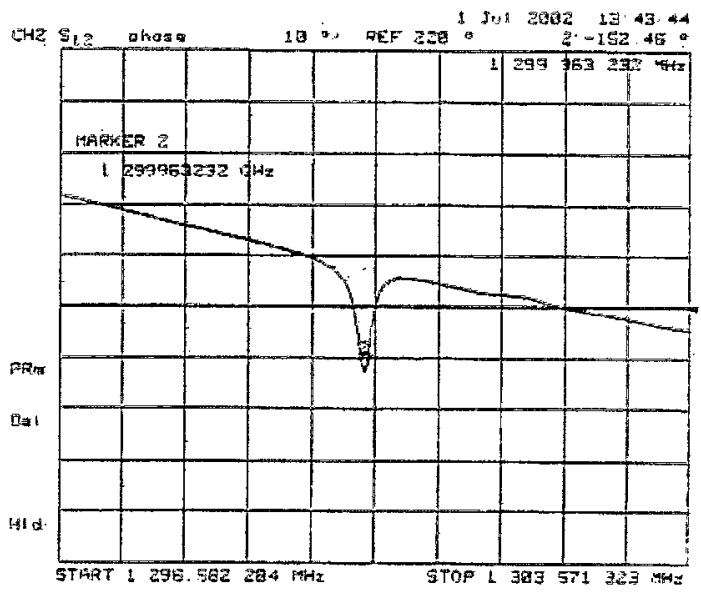

(d)

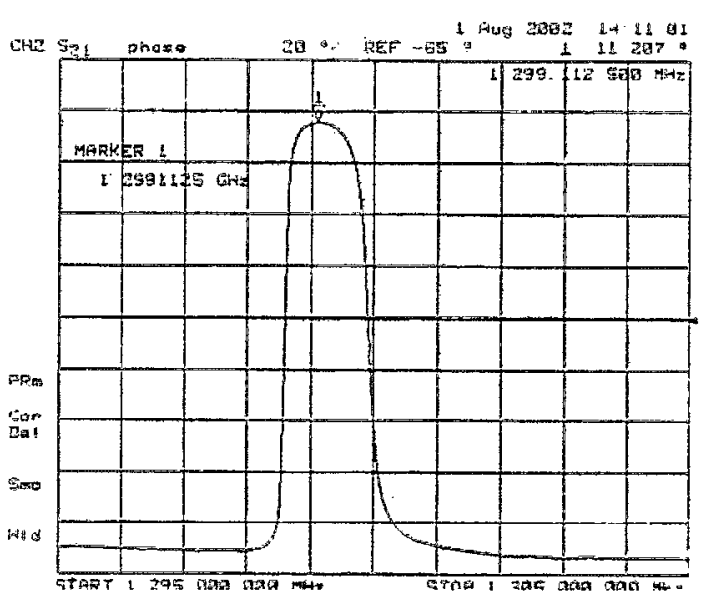

(f)

Fig.2. Some typical measured S21 responses of the cavity with different crosstalk. The left column displays the magnitudes of $\mathrm{S} 21$; and the right column, their phases. 
In order to make a better measurement at room temperature, we employed a third probe (probe $\mathrm{C}$ in Fig. 1) made of a semi-rigid coaxial cable, because it can be inserted close to the cavity to increase the coupling with less crosstalk. Fig. 2(e) and (f) show the results by using probe $\mathrm{C}$ and probe $\mathrm{A}$. The dip frequency shifts away from the peak that the resonant curve becomes meaningful.

The following simulation and analysis explain these phenomena.

\section{MODELING AND SIMULATION}

SUPERFISH has been used to simulate the cavity. ${ }^{[1]}$ However, it cannot simulate coupling, which is a 3-dimensional problem. One can employ a 3-D code such as MAFIA if the field analysis is of concern.

However, in the present case, the equivalent circuit model is more convenient and can be simulated by PSpice. Especially, it gives a clear physical concept to help direct the design. The cavity can be represented by an L-C-R resonant circuit. Its equivalent parameters can be deduced from the output of SUPERFISH as:

$$
\begin{aligned}
& \mathrm{C}_{0}=1.048 \mathrm{pF} \\
& \mathrm{L}_{0}=14.30 \mathrm{nH} \\
& \mathrm{R}_{0}=9.34 \mathrm{e} 5 \mathrm{ohm} \text { (room temp) } \\
& \mathrm{R} / \mathrm{Q}=116.8 \mathrm{ohm}
\end{aligned}
$$

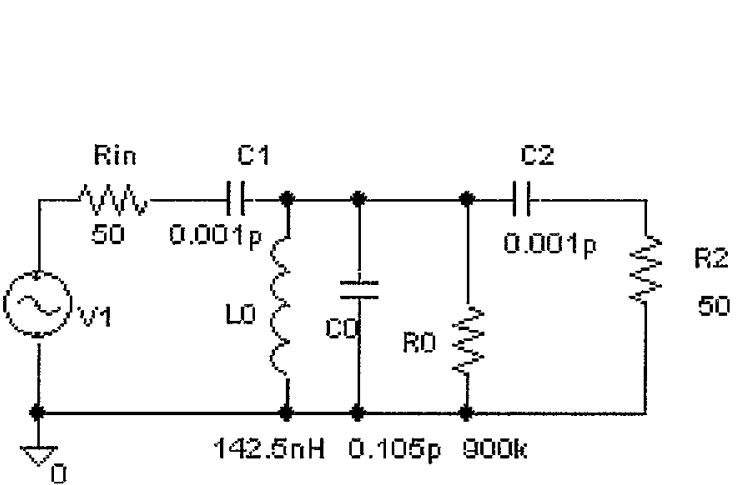

(a)

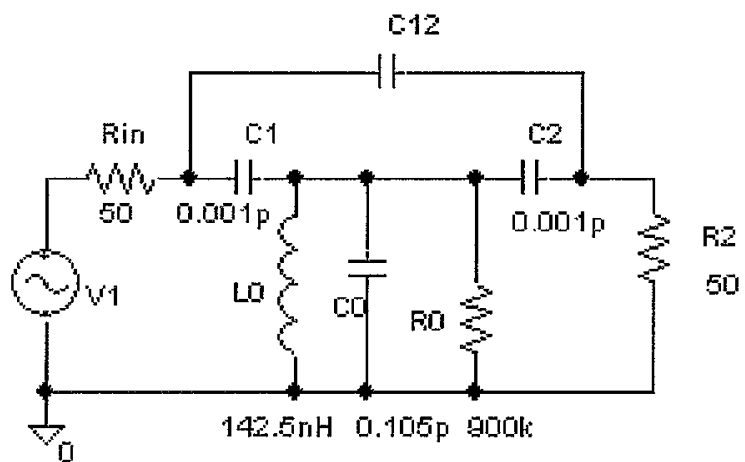

( b )

Fig. 3. Equivalent circuit of the cavity and couplers (a), with crosstalk added (b)

Fig. 3(a) shows the equivalent circuit, where $\mathrm{C}_{0}-\mathrm{L}_{0}-\mathrm{R}_{0}$ represents the cavity. $\mathrm{C} 1$ and C2 represent the input and pickup couplers, respectively, with each connected to a 50 ohm cable. The values are arbitrarily defined for the moment. A capacitance $\mathrm{C} 12$ is added in Fig. 3(b). It represents a direct coupling between input and pickup and introduces crosstalk.

Here all the couplings are attributed to capacitance, because the probes are of rod shape and located in the electric field area. This may not always be true, as mentioned above, if the pickup is a loop. The latter case is complicated and will be dealt with in the next section.

Fig. 4 shows the results from PSpice. Evidently, without direct coupling, the curves 
display normal resonance. The phase changes 180 degrees when the frequency crosses the resonance. When $\mathrm{C} 12$ is added ( $\mathrm{C} 12=0.001 \mathrm{pF}$ is chosen in this curve), both the magnitude and phase display are distorted, exactly as we measured.

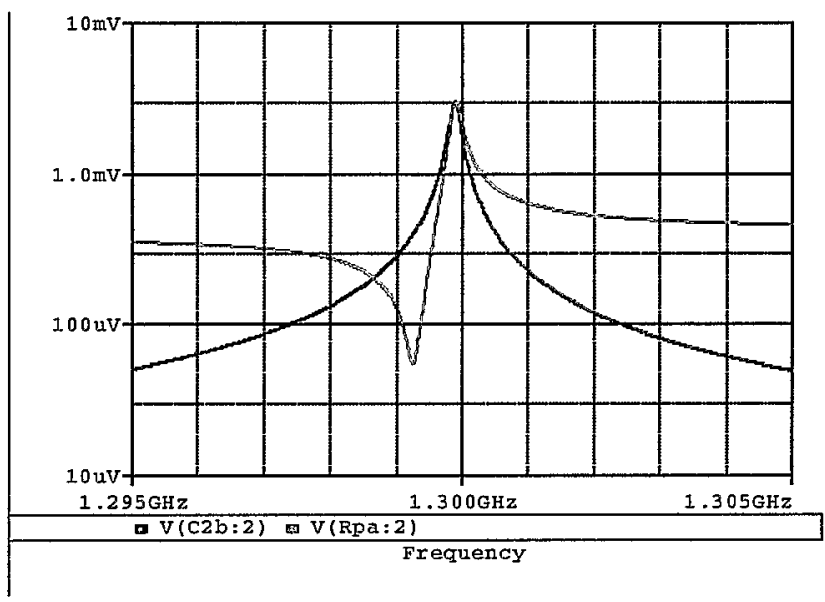

( a ) Magnitude of S21

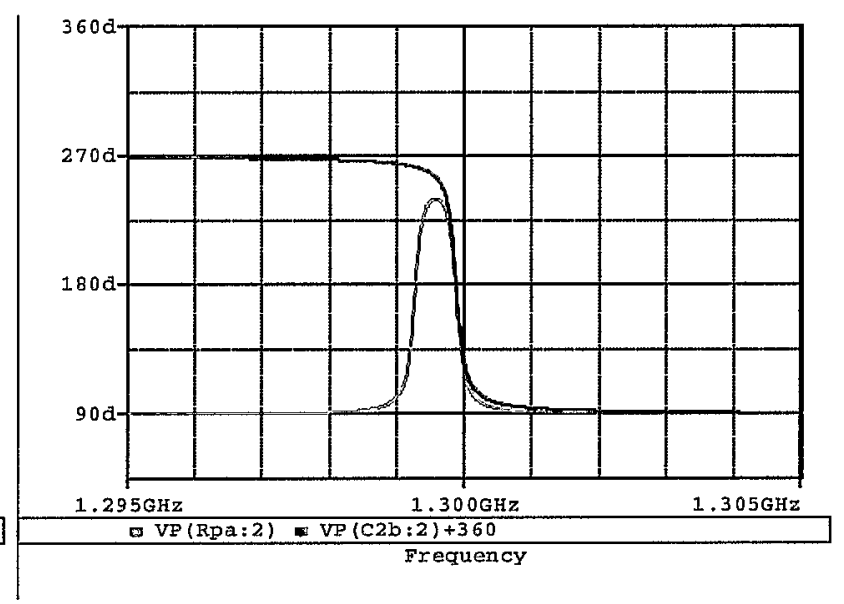

( b ) Phase of S21

Fig. 4. The simulation results of circuits Fig. 3(a)--green, and Fig.3(b)--red.

The dip frequency is due to the interference of two signals. One is the normal coupling through the cavity, of which the phase is very frequency sensitive. The other is due to the direct coupling, which is not sensitive to frequency. When the two signals had opposite phases, the signals cancel each other and thus formed a dip.

If one increases the $\mathrm{Q}$ of the cavity by cooling it down from room temperature to its superconducting state, in the vicinity of the resonant frequency the coupled signal through the cavity becomes much stronger than that through $\mathrm{C12}$, and the crosstalk becomes less important. The response will revert to a normal resonance.
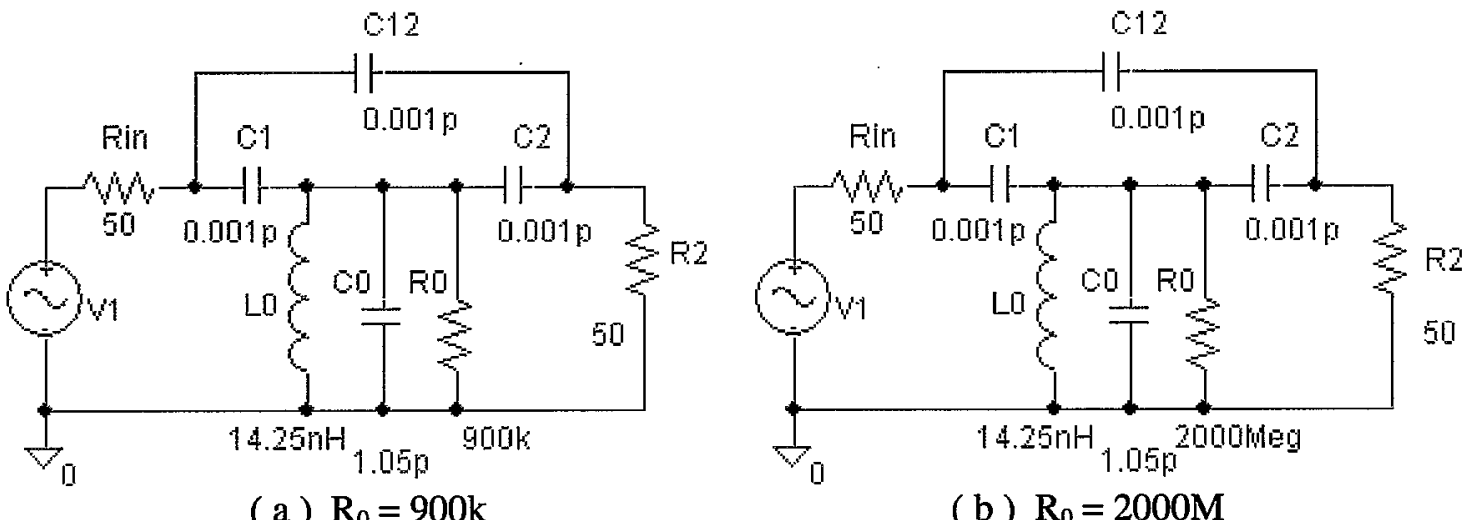

(b) $\mathrm{R}_{0}=2000 \mathrm{M}$

Fig. 5. The same direct coupling but different cavity $Q$

Fig. 5 shows the simulated circuit with (a) $R_{0}=900 \mathrm{k} \Omega$ and (b) $R_{0}=2000 \mathrm{M} \Omega$, representing room temperature and superconducting respectively. The PSpice output is shown in Fig. 6: the peak is much sharper. Meanwhile, the phase changes 180 degree 
when the frequency crosses the resonance, although the direct coupling (C12) remains the same.

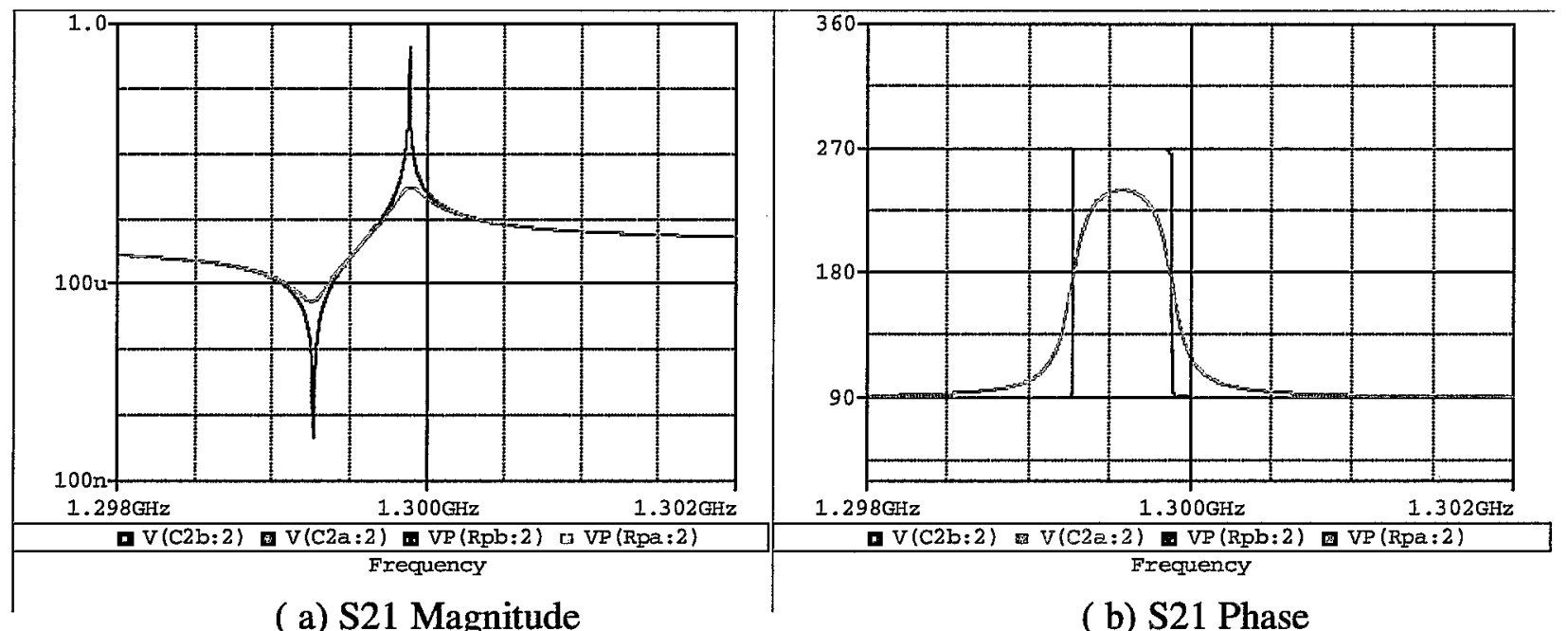

Fig. 6. S21 of different $Q$ value, results of Fig. 5(a )--red and Fig.5(b)--green.

This verifies the above argument that the crosstalk becomes less important when the cavity is superconducting.

To check the effect of the direct coupling, Fig. 7 shows the response with different values of $\mathrm{C} 12$.

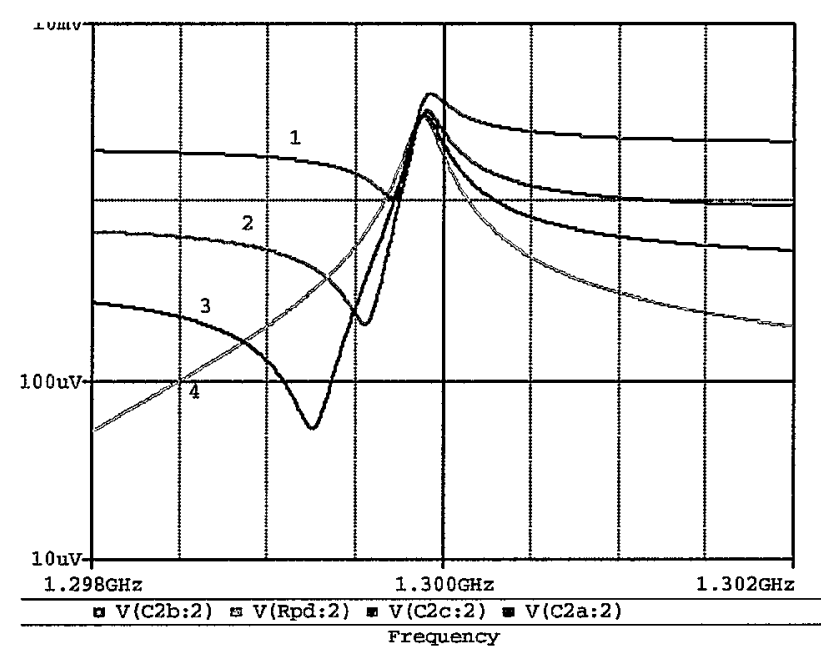

(a) S21 magnitude

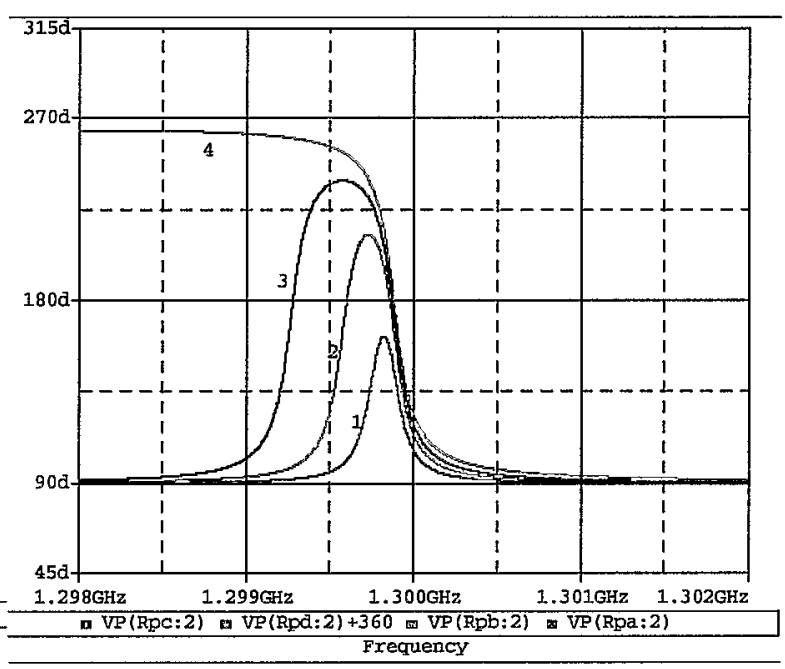

( b ) S21 phase

Fig. 7. S21 response with different values of $\mathrm{C} 12$ :

$$
\text { (1 -- 0.005pF, } 2 \text {-- 0.002pF, } 3 \text {-- 0.001pF, } 4-0.0002 \mathrm{pF} \text { ) }
$$

It shows that the weaker the direct coupling (the smaller C12), the larger the frequency separation between peak and dip, also the larger the phase expansion. When it is weak enough, as in curve 4, the dip frequency is too far away to be evident in the figure, meaning that the crosstalk is negligible. Meanwhile, the phase expanded to 180 
degrees.

If the direct coupling is very strong, then not only will the resonant curve be distorted, but also the magnitude of S21 will increase and the peak frequency shift to a higher one.

\section{ANALYSIS}

The simulation gives a clear response, but doesn't give an explicit relationship between its parameters. Therefore, it is necessary to analyze the equivalent circuit. Fig. 8 shows the model in question. Fig. 9 gives a generalized form, which resembles a bridge circuit.

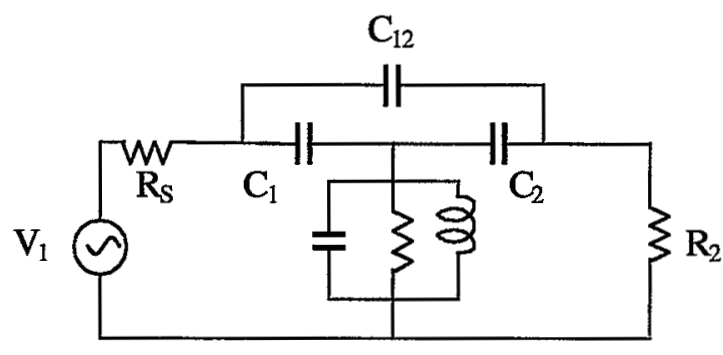

$\begin{array}{lll}\mathbf{C}_{\mathrm{C}} & \mathbf{R}_{\mathrm{C}} & \mathrm{L}_{\mathrm{C}}\end{array}$

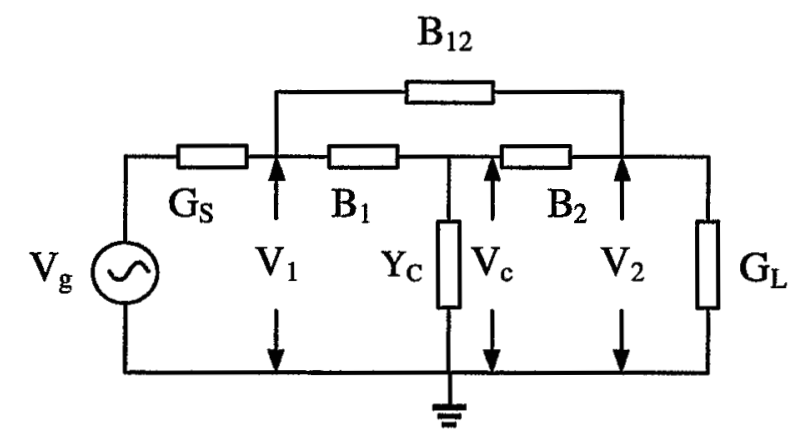

Fig.8. The equivalent circuit of the cavity and its couplers

Fig. 9. The generalization of Fig.8

Applying Kirchhoff law, after algebraic manipulation one obtains the following matrix equation.

$$
\left(\begin{array}{ccc}
Y_{1} & -B_{12} & -B_{1} \\
-B_{12} & Y_{2} & -B_{2} \\
-B_{1} & -B_{2} & Y_{0}
\end{array}\right)\left(\begin{array}{c}
V_{1} \\
V_{2} \\
V_{C}
\end{array}\right)=\left(\begin{array}{c}
G_{S} \\
0 \\
0
\end{array}\right) V_{g},
$$

where $\quad Y_{1}=G s+B_{1}+B_{12}$

$$
\begin{aligned}
& Y_{2}=G_{L}+B_{2}+B_{12} \\
& Y_{O}=Y c+B_{1}+B_{2} .
\end{aligned}
$$

$Y_{O}$ is the total admittance of the cavity including the coupling capacitance. In our special case, $\mathrm{G}_{\mathrm{S}}=\mathrm{G}_{\mathrm{L}}=\mathrm{G}_{0}=1 / 50(\mathrm{ohm})$, and the couplings are very weak such that $B_{l}$, $B_{2}, B_{12}$ are negligible in comparison with $G_{0}$. Then approximately,

$$
Y_{1}=Y_{2}=G_{0}=0.02 \text { mho }
$$

Solving the equation (1), one obtains:

$$
\frac{V_{2}}{V_{g}}=\frac{Y_{0} B_{12}+B_{1} B_{2}}{G_{0} Y_{0}-B_{1}^{2}-B_{2}^{2}} .
$$

The coupling coefficient $\beta$ related to the coupling capacitance can be deduced by the ratio of power loss inside and outside the cavity in the absence of crosstalk. From the 
parameters in Fig. 8 one obtains the following formulas.

$$
\begin{aligned}
\beta_{2} & =\frac{P_{L}}{P_{C}}=\frac{V_{C}^{2}\left(R_{2} \omega_{0} C_{2}\right)^{2} / 2 R_{2}}{V_{C}^{2} / 2 R_{C}}=R_{2} R_{C}\left(\omega_{0} C_{2}\right)^{2} \\
& =\frac{R_{C} R_{2}}{L_{C} C_{C}} C_{2}^{2}=Q_{0}^{2} \frac{R_{2}}{R_{C}} \frac{C_{2}^{2}}{C_{C}^{2}}
\end{aligned}
$$

Similarly $\quad \beta_{1}=Q_{0}^{2} \frac{R_{S}}{R_{C}} \frac{C_{1}^{2}}{C_{C}^{2}}$.

Note that $R_{S}=R_{2}=1 / G_{0}$, and $R_{c} \omega_{b} C_{c}=Q_{0}$.

Now we can explore the properties of formula (4). Only $Y_{0}$ is frequency sensitive. The other parameters can be regarded as approximately constant. In general, a ratio of polynomials can be characterized by its poles and zeros. Without crosstalk (i.e. $B_{12}=0$ ), the numerator in formula (4) becomes constant and thus has no zero, corresponding to a simple resonance.

With crosstalk one needs to substitute the parameters in Fig.8:

$$
B_{1}=j \omega C_{1}, \quad B_{2}=j \omega C_{2}, \quad B_{12}=j \omega C_{12} .
$$

After tedious algebraic manipulation that formula (4) can be rewritten in the form:

$$
\frac{V_{2}}{V_{g}}=\frac{j \omega C_{12}}{G_{0}(1+\beta)} \cdot \frac{1+j Q_{z} \cdot 2\left(f-f_{z}\right) / f_{0}}{1+j Q_{L} \cdot 2\left(f-f_{0}\right) / f_{0}},
$$

where

$$
\begin{aligned}
& \beta=\beta_{1}+\beta_{2} \quad\left(\beta=\beta_{1}+\beta_{2}+\beta_{3} \text { if there are } 3 \text { probes }\right) \\
& Q_{L}=\frac{Q_{0}}{1+\beta}, \\
& Q_{Z}=Q_{o}\left(1+\delta_{Z}\right), \\
& f_{Z}=f_{0}\left(1-\frac{1}{2} \delta_{Z}\right),
\end{aligned}
$$

and

$$
\delta_{Z}=\frac{C_{1} C_{2}}{C_{0} C_{12}} .
$$

There is a pole at $f=f_{0}$, and a zero at $f=f_{Z}$. This verifies what we have observed in the measurement. Around the resonant frequency $f_{0}$, the $Q$ is $Q_{L}$. Around $f_{Z}$, the dip shows an anti-resonance with $Q$ of $Q_{Z}$. However, if the crosstalk is serious, $f_{0}$ is close to $f_{Z}$, and the resonant curve is so distorted that the peak frequency $f_{\max }$ is not exactly at $f_{0}, f_{\min }$ is not exactly at $f_{Z}$, and the measured $Q$ from $3 \mathrm{~dB}$ bandwidth is not exact $Q_{L}$.

Here we have introduced the important parameter $\delta_{Z}$, relating the relative frequency separation of $f_{Z}$ and $f_{o}$. (see (7e))

$$
\delta_{Z}=\frac{2\left(f_{0}-f_{Z}\right)}{f_{0}}
$$


The closer the two frequencies, the more the distortion of the resonant curve, implying more serious crosstalk.

In order to give a clear physical meaning to $\delta_{Z}$, let's rewrite (7f) as:

$$
\delta_{z}=\frac{\left(C_{1} / C_{0}\right)\left(C_{2} / C_{0}\right)}{\left(C_{12} / C_{0}\right)}
$$

The numerator is a measure of the coupling through the cavity, while the denominator is a measure of the direct coupling. Thus the ratio gives a measure of the interference. The larger $\mathrm{C} 12$, the smaller $\delta_{\mathrm{Z}}$, and the stronger the crosstalk.

An implicit fact for forms (7) and (8) is that all the couplings are capacitive, as displayed by Fig.(8). Consequently, $\delta_{Z}$ is positive. However, it is not required by formula (4).

If the pickup coupling is primarily magnetic, then $B_{2}=j \omega C_{2}$ in (6) will be replaced by $B_{2}=-j \omega C_{2}=-j /(\omega L)$; namely the sign is changed. (The symbol $C$ or $L$ is not important, because it's only a constant in the vicinity of the resonant frequency; only the sign is important.) In this case $\delta_{Z}$ is negative. Then from (7e), $f_{Z}>f_{0}$; i.e. the dip frequency is higher than the peak frequency. Meanwhile, the phase of S21 changes sign too (See later for detail.). This explains the phenomena observed in Figs. 2(c) and (d).

In addition, from formulas (7a), (7c) and (7d) one can see that $Q_{L}<Q_{0}$, while $Q_{Z}>$ $Q_{0}$. This implies that the dip (or anti-resonance) is sharper than the peak, agreeing with what we've observed.

The agreement with observation once again verifies the circuit model. In order to get more general and convenient formulas, we can make a further normalization:

$$
\begin{aligned}
& \delta=\frac{2\left(f-f_{0}\right)}{f_{0}}, \\
& F=Q_{o} \delta,
\end{aligned}
$$

and

$$
F_{Z}=Q_{o} \delta_{Z} \text {. }
$$

Each has a physical meaning. $\delta$ is the relative frequency deviation. $F$ is the normalized frequency deviation, such that $F=1$ corresponds to a frequency at the edge of the 3-dB bandwidth. $F_{Z}$ is the normalized crosstalk parameter that measures the deviation of the dip frequency from the resonant frequency, It gives a good measure of the crosstalk.

Substituting (5), (7f) and (10), then (7a) can be rewritten as:

$$
\frac{V_{2}}{V_{g}}=\frac{j \sqrt{\beta_{1} \beta_{2}}}{1+\beta} \cdot \frac{1}{1+j k_{L} F} \cdot \frac{1+j k_{Z}\left(F+F_{z}\right)}{F_{Z}}
$$

where $k_{Z}$ and $k_{L}$ are constants very close to unity:

$$
k_{Z}=1+\delta_{Z} \approx 1,
$$

and

$$
k_{L}=1 /(1+\beta) \approx 1 \text {. }
$$


Equation (11) has a clear physical meaning. On the RHS, the first two fractions represent the response in the absence of direct coupling. The first one is the magnitude of the coupling at resonant frequency. The second manifests the resonant performance. The third fraction involving $F_{Z}$ manifests the effect of the direct coupling or the crosstalk. It approaches unity for $F_{Z} \rightarrow \infty$, i.e. whenever either $Q_{0}$ or $\delta_{Z}$ is very large. This verifies that $F_{Z}$ is an even better measure of crosstalk than the parameter $\delta_{Z}$.

At room temperature, $F_{Z}$ is in a range that the crosstalk must be taken into account. When cooled down to the superconducting state, $Q_{0}$ increases a few orders of magnitude, such that the condition $F_{Z} \gg 1$ is realized, and the crosstalk vanishes.

\section{THE RELATIONSHIP TO THE TESTED PARAMETERS}

Using the analyses above, we now are ready to calculate the parameters we want from the parameters that we can measure.

The parameters we want to know are the resonant frequency $f_{0}, Q_{0}$, coupling coefficient $\beta$, and external $Q$. Without crosstalk, for a simple resonator, they can easily be determined by the network analyzer. But, for the case concerned, neither can be measured directly without significant error.

The measurable parameters are S11 and S21, with corresponding frequencies. (see Fig.12).

Let's recall briefly the measurement of the coupling coefficient $\beta$ of a normal cavity. It's simply determined by the SWR at the input transmission line; it's a function of frequency. (Using symbol $\rho=\mathrm{SWR}$ for short..)

or

$$
\begin{array}{ll}
\beta=\rho\left(f_{0}\right) & \text { (over-coupling), } \\
\beta=1 / \rho\left(f_{0}\right) & \text { (under-coupling). }
\end{array}
$$

In our case, coupling is designed to be close to critical $(\beta=1)$ in the when in superconducting state, while at room temperature, $\beta$ is of the order of $10^{-4}$. In this case, the SWR is of the order of $10^{4}$, which is too high to measure accurately. The SWR looks like Fig. 10. The SWR at off-resonance is only about 17, though in principle, for a lossless transmission line, the SWR is infinite.

The finite SWR implies the existence of a residual loss somewhere in the transmission line. Assume an equivalent lumped resistance $R_{r}$ at the entrance of the cavity, as shown in Fig. 11. At off-resonance there is no power entering the cavity, but it is not a perfect open circuit due to residual losses. Then the measured SWR is

$$
\rho_{l}=R_{r} .
$$

At resonance the cavity will introduce a minor extra loss, whereupon the SWR is

$$
\rho_{2}=\frac{R_{r} R_{c}}{R_{r}+R_{c}}
$$

One can easily find the $\beta$, which equals the SWR due to the cavity only: 


$$
\beta=\frac{1}{R_{c}}=\frac{\rho_{1}-\rho_{2}}{\rho_{1} \rho_{2}}
$$

This formula gives a rough estimation of very weak coupling. However, the precision is not satisfactory, due to the error of the measured $\rho s$.

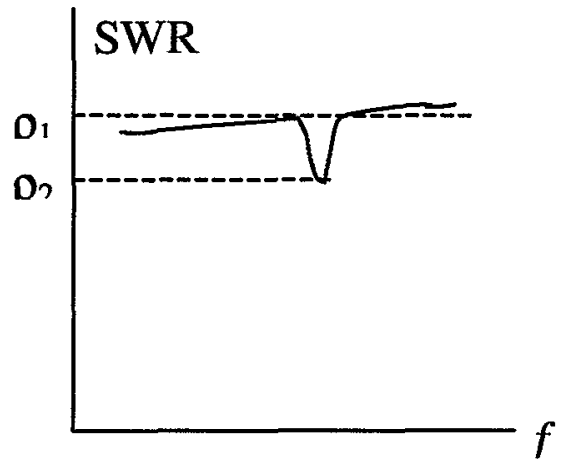

Fig. 10 A measured SWR as a function of frequency

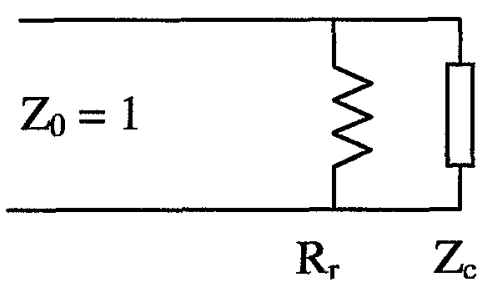

Fig. 11 An equivalent circuit of a lossy transmission line. Zc represents cavity impedance that $\mathrm{Zc}\left(\mathbf{f}_{0}\right)=\mathrm{Rc}$.

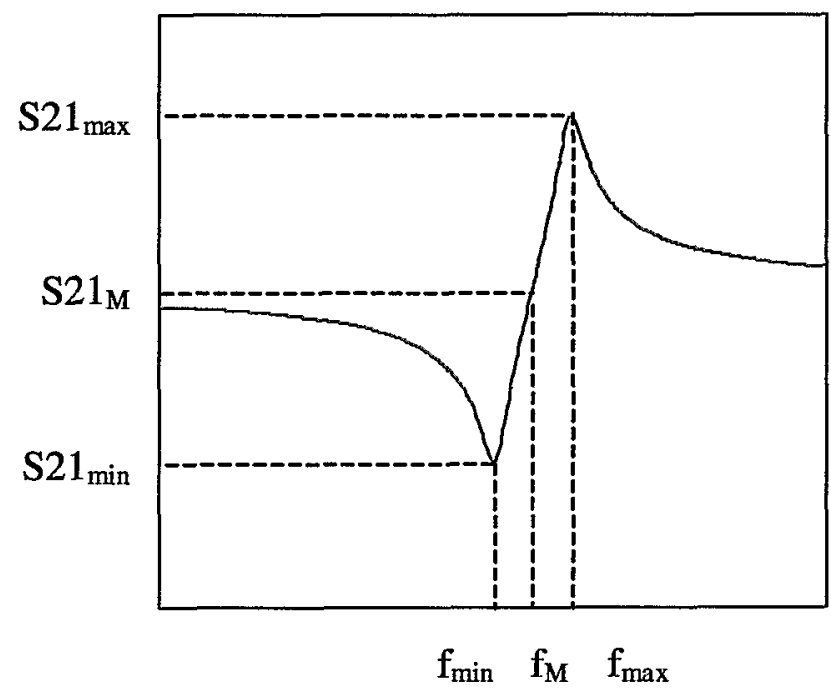

Fig. 12 The measurable parameters

We should rely mostly on the measurement of $S 21$.

Note that $V g$ in the equivalent circuit Fig.9 is twice the injection voltage on the input transmission line, or $2 V_{+}$; the scattering parameter $\mathrm{S} 21$ is

$$
S 21=\frac{V_{2}}{V_{+}}=\frac{2 V_{2}}{V_{g}} .
$$

In a network analyzer, S21 usually reads in $\mathrm{dB}$ for magnitude and phase. The magnitude is: 


$$
S 21=20 \log \left|\frac{V_{2}}{V_{+}}\right|=20 \log \left|\frac{2 V_{2}}{V_{g}}\right|(\mathrm{dB}) .
$$

From (11) we obtain:

$$
S 21=10 \log \left\{\frac{4 \beta_{1} \beta_{2}}{1+\beta_{1}+\beta_{2}} \cdot \frac{1}{1+k_{L}^{2} F^{2}} \cdot \frac{1+k_{z}^{2}\left(F+F_{z}\right)^{2}}{F_{z}^{2}}\right\} .
$$

The phase is:

$$
\Phi(S 21)=\arctan \left[k_{z}\left(F+F_{z}\right)\right]-\arctan \left(k_{L} F\right) .
$$

Note that both $k_{L}$ and $k_{Z}$ are very close to unity and thus negligible in most cases. $\beta=$ $\beta_{1}+\beta_{2} \ll 1$, also is negligible in many cases. Obviously, if crosstalk is negligible (i.e., $F_{\mathrm{Z}}>>1$ ), the maximum $\mathrm{S} 21$ occurs at resonance or $F=0$, one obtains

$$
\mathrm{S} 21_{\max }=10 \log \left(4 \beta_{1} \beta_{2}\right) .
$$

This well-known formula applies in the absence of crosstalk.

However, if the crosstalk is not negligible, the last two fractions in (18) must be taken into account. The maximum S21 does not occur at resonant frequency. Differentiating (18) with respect to $F$, one can find the peak and dip frequencies to be:

$$
F_{\max }=\frac{\sqrt{F_{z}^{2}+4}-F_{z}}{2},
$$

and $\quad F_{\min }=-\frac{\sqrt{F_{z}^{2}+4}+F_{z}}{2}$,

where $F_{\max }$ and $F_{\min }$ are normalized frequencies as defined in (10), or

$$
\text { and } \quad \begin{aligned}
& F_{\max }=Q_{0} \cdot 2\left(f_{\max }-f_{0}\right) / f_{o}, \\
& F_{\min }=Q_{0} \cdot 2\left(f_{\min }-f_{0}\right) / f_{0} .
\end{aligned}
$$

Note that $F_{\max }$ is always positive, meaning the peak frequency is not equal to the resonant frequency $f_{0}$ (pole point) except when crosstalk is negligible $\left(F_{Z} \gg 1\right)$. This deviation is

$$
f_{\max }-f_{0}=\frac{f_{0}}{2 Q_{0}} \cdot \frac{\sqrt{F_{z}^{2}+4}-F_{z}}{2} .
$$

This deviation was found to be up to $70 \mathrm{kHz}$ in some cases. Similarly, the dip frequency $f_{\text {min }}$ also deviates from the "zero point" frequency $f z$.

As seen in Fig. 12, $f_{\max }$ and $f_{\min }$ are measurable. From (22) and (21) their difference can be used to determine the crosstalk parameter $F_{Z}$, providing $Q_{o}$ is known.

$$
\begin{aligned}
& F_{z}^{\prime}=F_{\max }-F_{\min }=Q_{0} \cdot 2\left(f_{\max }-f_{\min }\right) / f_{0}, \\
& F_{Z}=\sqrt{F_{z}^{2}-4} .
\end{aligned}
$$


Substituting (21) into (18), $S 21_{\max }$ and $S 21_{\min }$ are found to be:

and

$$
\begin{aligned}
& S 21_{\max }=10 \cdot \log \left(\frac{4 \beta_{1} \beta_{2}}{1+\beta} \cdot \frac{1}{F_{Z}^{2}} \cdot \frac{\sqrt{F_{Z}^{2}+4}+F_{Z}}{\sqrt{F_{Z}^{2}+4}-F_{Z}}\right), \\
& S 21_{\min }=10 \cdot \log \left(\frac{4 \beta_{1} \beta_{2}}{1+\beta} \cdot \frac{1}{F_{Z}^{2}} \cdot \frac{\sqrt{F_{Z}^{2}+4}-F_{Z}}{\sqrt{F_{Z}^{2}+4}+F_{Z}}\right) .
\end{aligned}
$$

In practice, the signal $S 21_{\min }$ is usually too weak to be reliable. Instead, one can measure a middle value $\mathrm{S} 21_{\mathrm{M}}$ at the middle frequency $f_{M}$, i.e.

$$
f_{M}=\frac{f_{\max }+f_{\min }}{2}, \quad F_{M}=\frac{F_{\max }+F_{\min }}{2}=\frac{F_{Z}}{2}
$$

and

$$
S 21_{M}=10 \cdot \log \left(\frac{4 \beta_{1} \beta_{2}}{1+\beta} \cdot \frac{1}{F_{Z}^{2}}\right)
$$

Then

$$
\Delta S_{0 M}=S 21_{\max }-S 21_{M}=10 \cdot \log \frac{\sqrt{F_{Z}^{2}+4}+F_{Z}}{\sqrt{F_{Z}^{2}+4}-F_{Z}}
$$

Defining $\quad C=10^{\Delta S_{0 M} / 20}$,

$\Delta \mathrm{S}_{0 \mathrm{M}}$ is a measured quantity, so $C$ is a measurable parameter related to crosstalk From (28) we have

$$
C=\frac{\sqrt{F_{Z}^{2}+4}+F_{Z}}{2}, \quad \text { and } \quad C^{2}=\frac{\sqrt{F_{Z}^{2}+4}+F_{Z}}{\sqrt{F_{Z}^{2}+4}-F_{Z}} .
$$

Solving the equation, one can find $F_{Z}$ from the measurable parameter $\Delta S_{O M}$ and $C$ :

$$
F_{Z}=C-\frac{1}{C}
$$

According to (25a), (27) and (28), the approximate (20) should be corrected as

where

$$
\begin{aligned}
& S 21_{\max }=10 \log \left(4 \beta_{1} \beta_{2}\right)+\Delta S_{0}, \\
& \Delta S_{0}=10 \cdot \log \left(\frac{1}{F_{Z}^{2}} \cdot \frac{\sqrt{F_{Z}^{2}+4}+F_{Z}}{\sqrt{F_{Z}^{2}+4}-F_{Z}}\right)=10 \cdot \log \frac{C^{2}}{F_{Z}^{2}} \\
& \quad=-20 \cdot \log \left(1-\frac{1}{C^{2}}\right)
\end{aligned}
$$

From $\mathrm{S} 21_{\max }$ one can easily find the product $\beta_{1} \beta_{2}$. We have to employ three probes, as shown in Fig.1. We can make different combinations, such that two of them connect to the two ports of the network analyzer, and measure the $\mathrm{S} 21$ for each combination. Once one measures the three products $\beta_{A} \beta_{B}, \beta_{A} \beta_{C}$, and $\beta_{C} \beta_{B}$, it is easy to find each individual 
$\beta$ value by

$$
\beta_{A}=\sqrt{\frac{\left(\beta_{A} \beta_{B}\right) \cdot\left(\beta_{A} \beta_{C}\right)}{\left(\beta_{C} \beta_{B}\right)}}
$$

As mentioned before, the phase of S21 displays a stroke when crosstalk is not negligible. The magnitude of the phase stroke can be found by seeking the maximum of the $\Phi(\mathrm{S} 21)$ from (19). Differentiating $\Phi(\mathrm{S} 21)$ with respect to $F$, one finds that $\Phi_{\max }$ occurs at

$$
F=F_{Z} / 2 \quad \text { (the same frequency as } \mathrm{F}_{\mathrm{M}} \text { ) }
$$

and $\Phi_{\max }=2 \cdot \arctan \frac{F_{Z}}{2}$,

or $\quad F_{Z}=2 \cdot \tan \frac{\Phi_{\max }}{2}$.

As $\Phi_{\max }$ is measurable, it gives another way to determine $F_{Z}$. But, it is suitable only if $F_{Z}$ is quite small. Formulas (24), (30) and (35) show three ways to determine $F_{Z}$. Formula (30) is the best. The rest can be used for cross checks.

Now let's find the deviation of the $Q$ value. From (18) and (22), one can find the 3$\mathrm{dB}$ bandwidth. Considering only the factors depending on frequency $F$ in (18), the frequency at the edge of bandwidth is:

$$
\frac{1+\left(F+F_{z}\right)^{2}}{1+F^{2}}=\frac{1}{2} \cdot \frac{1+\left(F_{\max }+F_{z}\right)^{2}}{1+F_{\max }^{2}} .
$$

This equation can be simplified as:

$$
\frac{2 F F_{z}+F_{z}^{2}}{1+F^{2}}=p, \quad \text { or } \quad p F^{2}-2 F F_{z}-\left(F_{z}^{2}-p\right)=0
$$

where $\quad p=\frac{1}{2} \cdot \frac{1+\left(F_{\max }+F_{z}\right)^{2}}{1+F_{\max }^{2}}-1$.

It turns out:

$$
F_{3,4}=\frac{F_{z} \pm \sqrt{F_{z}^{2}+p\left(F_{z}^{2}-p\right)}}{p} .
$$

The normalized bandwidth is

$$
F_{3}-F_{4}=\frac{2 \sqrt{F_{z}^{2}+p\left(F_{z}^{2}-p\right)}}{p} .
$$

The real $\mathrm{Q}$ is that in the case of no crosstalk, i.e. $F_{z} \gg 1, p=\left(F_{z}^{2}-1\right) / 2, F_{3}-F_{4}=2$. The case $F_{3}-F_{4}>2$ implies that $\mathrm{Q}$ decreases. Therefore, the ratio of the real $\mathrm{Q}$ and the measured $Q$ is:

$$
G_{q}=\frac{\sqrt{F_{z}^{2}+p\left(F_{z}^{2}-p\right)}}{p} .
$$


Substituting (21),(30) and (38), after algebraic manipulation one obtains

$$
G_{q}=\sqrt{2\left(\frac{C^{2}-1}{C^{2}-2}\right)^{2}-1} .
$$

Thus the real loaded $\mathrm{Q}$ is

$$
Q_{L}=G_{q} Q_{t e s t e d} .
$$

\section{RESULTS OF MEASUREMENT}

We tested a superconducting cavity made of niobium, with both launcher and pickup of different sizes. Also employed was a probe C (see Fig.1), which is an axial semi-rigid cable with the tip stripped, such that it forms an antenna. The sizes of the probes are shown in Table 1. Selected measured data are in Table 2. Also shown are the deduced data calculated by means of formulas derived above. Table 3 summarizes the final coupling coefficients and the corresponding external Q. Figs.13-16 show the curves.

\begin{tabular}{|c|c|c|c|c|c|}
\hline \multicolumn{3}{|c|}{ Probe A (Launcher) } & \multicolumn{3}{|c|}{ Probe B (Pick-up) } \\
\hline ID & $\begin{array}{l}\text { Length } \\
\text { (inch) }\end{array}$ & $\begin{array}{l}\text { Length } \\
\text { (mm) }\end{array}$ & ID & $\begin{array}{l}\text { Length } \\
\text { (inch) }\end{array}$ & $\begin{array}{l}\text { Length } \\
\text { (mm) }\end{array}$ \\
\hline$\overline{\mathrm{A} 1}$ & 2.651 & 67.34 & $\overline{\text { B1 }}$ & 2.184 & 55.47 \\
\hline $\mathrm{A} 1 \mathrm{~b}$ & 2.532 & 64.31 & B2 & 1.934 & 49.12 \\
\hline Alc & 2.413 & 61.29 & B3 & 1.684 & 42.77 \\
\hline A1d & 2.248 & 57.10 & B4 & 1.434 & 36.42 \\
\hline A2 & 2.247 & 57.07 & & & \\
\hline A3 & 2.172 & 55.17 & & & \\
\hline
\end{tabular}

Table 1 Sizes of probes

\begin{tabular}{|c|c|c|c|c|c|c|c|}
\hline Port 1_probe & A1a & Ala & $\mathrm{C}$ & $\mathrm{Alb}$ & Alc & Ald & B2 \\
\hline Port 2_probe & $\mathrm{C}$ & B1 & B1 & C & $\mathrm{C}$ & $\mathrm{C}$ & C \\
\hline$f_{0}(\max )$ & $\begin{array}{c}1299.825 \\
272\end{array}$ & $\begin{array}{c}1299.868 \\
750\end{array}$ & $\begin{array}{c}1299.812 \\
716\end{array}$ & $\begin{array}{c}1299.818 \\
750\end{array}$ & $\begin{array}{c}1299.812 \\
500\end{array}$ & $\begin{array}{c}1299.812 \\
500\end{array}$ & $\begin{array}{c}1299.812 \\
500\end{array}$ \\
\hline $\mathrm{Q}_{\mathrm{L}}$ & 6439.8 & - & 6520.3 & 6432.5 & 6453.4 & \begin{tabular}{|l|}
6444.8 \\
\end{tabular} & 6492.4 \\
\hline$f_{z}(\min )$ & $\begin{array}{c}1298.375 \\
000 \\
\end{array}$ & \begin{tabular}{|c}
1299.646 \\
875 \\
\end{tabular} & -- & $\begin{array}{c}1298.337 \\
500 \\
\end{array}$ & $\begin{array}{c}1298.131 \\
250 \\
\end{array}$ & \begin{tabular}{|c|}
1298.362 \\
500 \\
\end{tabular} & $\begin{array}{c}1298.071 \\
875\end{array}$ \\
\hline$f_{M}$ & $\begin{array}{c}1299.100 \\
640\end{array}$ & $\begin{array}{c}1299.757 \\
810\end{array}$ & -- & $\begin{array}{c}1299.078 \\
125\end{array}$ & $\begin{array}{c}1298.971 \\
875 \\
\end{array}$ & $\begin{array}{c}1299.087 \\
500\end{array}$ & $\begin{array}{c}1298.942 \\
187\end{array}$ \\
\hline $\mathbf{S} 21\left(\mathrm{f}_{0}\right) \mathrm{dB}$ & -46.864 & -68.449 & -54.532 & -49.234 & -51.636 & -54.954 & -59.827 \\
\hline $\mathrm{S} 21\left(\mathrm{f}_{\mathrm{z}}\right) \mathrm{dB}$ & -97.504 & -76.65 & -- & -93.084 & -98.164 & -98.142 & -106.02 \\
\hline $\mathrm{S} 21\left(\mathrm{f}_{\mathrm{M}}\right) \mathrm{dB}$ & -70.001 & -73.034 & - & -72.544 & -76.28 & -78.122 & -84.548 \\
\hline$\phi \max$ & - & 51.4 & -- & - & - & -- & - \\
\hline$S\left(f_{0}\right)-S(f m)$ & 23.137 & 4.585 & $=$ & 23.31 & 24.644 & 23.168 & 24.721 \\
\hline parameter C & 14.350 & 1.695 & - & 14.639 & 17.069 & 14.401 & 17.221 \\
\hline $\mathrm{Fz}$ & 14.280 & 1.105 & - & 14.570 & 17.010 & 14.332 & 17.163 \\
\hline $\mathrm{f}_{0}-\mathrm{f}_{\mathrm{Z}}$ & 1.45000 & 0.22188 & -- & 1.48125 & 1.68125 & 1.45000 & 1.74062 \\
\hline
\end{tabular}

Table 2 Selected measured data and deduced data 


\begin{tabular}{|l|c|c|c|c|c|c|c|}
\hline$\delta \mathrm{z}=2\left(\mathrm{f}_{0}-\mathrm{f}_{\mathrm{Z}}\right) / 2$ & $2.231 \mathrm{E}-3$ & $3.413 \mathrm{E}-4$ & -- & $2.279 \mathrm{E}-3$ & $2.587 \mathrm{E}-3$ & $2.231 \mathrm{E}-3$ & $2.678 \mathrm{E}-3$ \\
\hline $\mathrm{D}_{\mathrm{so}}$ & $4.228 \mathrm{E}-2$ & $3.714 \mathrm{E} 0$ & 0 & $4.063 \mathrm{E}-2$ & $2.986 \mathrm{E}-2$ & $4.198 \mathrm{E}-2$ & $2.934 \mathrm{E}-2$ \\
\hline $\mathrm{S} 21_{0}-\mathrm{D}_{\mathrm{so}}$ & -46.906 & -72.163 & -54.532 & -49.275 & -51.666 & -54.996 & -59.856 \\
\hline$\beta_{1} \beta_{2}$ & $5.097 \mathrm{E}-6$ & $1.519 \mathrm{E}-8$ & $8.805 \mathrm{E}-7$ & $2.954 \mathrm{E}-6$ & $1.704 \mathrm{E}-6$ & $7.913 \mathrm{E}-7$ & $2.584 \mathrm{E}-7$ \\
\hline $\mathrm{G}_{\mathrm{q}}$ & $1.01 \mathrm{E} 0$ & $2.862 \mathrm{E} 0$ & $1 \mathrm{E} 0$ & $1.009 \mathrm{E} 0$ & $1.007 \mathrm{E} 0$ & $1.01 \mathrm{E} 0$ & $1.007 \mathrm{E} 0$ \\
\hline $\mathrm{Q}_{\mathrm{L}}$ & 6502.8 & - & 6521.6 & 6493.0 & 6497.9 & 6507.4 & 6536.4 \\
\hline$\Delta \mathrm{f}$ & $6.863 \mathrm{E}-3$ & $5.809 \mathrm{E}-2$ & -- & $6.728 \mathrm{E}-3$ & $5.77 \mathrm{E}-3$ & $6.839 \mathrm{E}-3$ & $5.719 \mathrm{E}-3$ \\
\hline $\mathrm{f}_{0}$ (corrected) & $1,299.818$ & $1,299.811$ & -- & $1,299.812$ & $1,299.807$ & $1,299.806$ & $1,299.807$ \\
\hline
\end{tabular}

Table 3. Coupling coefficients and external Q

\begin{tabular}{|c||c|c|c|c||c|c|c|}
\hline \multirow{2}{*}{$\begin{array}{c}\text { Probe } \\
\text { ID }\end{array}$} & \multicolumn{3}{c|}{ Coupling Coefficient } & \multicolumn{3}{c|}{ External Q -- Qex } \\
\hline A1 & T.date & Jul. 16 & Aug.2 & Aug.29 & Jul. 16 & Aug.2 & Aug.29 \\
\hline A1b & $\beta_{\mathrm{A} 1}$ & $2.973 \mathrm{e}-4$ & $3.089 \mathrm{E}-4$ & $2.965 \mathrm{e}-4$ & $2.22 \mathrm{E} 7$ & $2.01 \mathrm{E} 7$ & $2.20 \mathrm{E} 7$ \\
\hline A1c & $\beta_{\mathrm{A} 1 \mathrm{~b}}$ & & & $1.718 \mathrm{e}-4$ & & & $3.79 \mathrm{E} 7$ \\
\hline A1d & $\beta_{\mathrm{A} 1 \mathrm{~d}}$ & & & $9.913 \mathrm{e}-5$ & & & $6.57 \mathrm{E} 7$ \\
\hline A2 & $\beta_{\mathrm{A} 2}$ & & & $4.603 \mathrm{e}-5$ & & & $1.41 \mathrm{E} 8$ \\
\hline A3 & $\beta_{\mathrm{A} 3}$ & & $2.956 \mathrm{E}-5$ & & & $2.10 \mathrm{E} 8$ & \\
\hline B1 & $\beta_{\mathrm{B} 1}$ & $5.348 \mathrm{e}-5$ & $5.144 \mathrm{E}-5$ & $5.123 \mathrm{e}-5$ & $1.23 \mathrm{E} 8$ & $1.21 \mathrm{E} 8$ & $1.27 \mathrm{E} 8$ \\
& & & $4.401 \mathrm{E}-5$ & & & $1.41 \mathrm{E} 8$ & \\
\hline B2 & $\beta_{\mathrm{B} 2}$ & $1.316 \mathrm{e}-5$ & & $1.503 \mathrm{e}-5$ & $5.02 \mathrm{E} 8$ & & $4.33 \mathrm{E} 8$ \\
\hline B3 & $\beta_{\mathrm{B} 3}$ & $2.377 \mathrm{e}-6$ & & & $2.78 \mathrm{E} 9$ & & \\
\hline B4 & $\beta_{\mathrm{B} 4}$ & $3.744 \mathrm{e}-7$ & $2.539 \mathrm{E}-7$ & & $1.76 \mathrm{E} 10$ & $2.44 \mathrm{E} 10$ & \\
\hline C & $\beta_{\mathrm{C}}$ & $2.090 \mathrm{e}-2$ & $3.038 \mathrm{E}-2$ & $1.719 \mathrm{e}-2$ & $3.61 \mathrm{E} 5$ & $2.00 \mathrm{E} 5$ & $3.79 \mathrm{E} 5$ \\
& & & $2.665 \mathrm{E}-2$ & & & $2.33 \mathrm{E} 7$ & \\
\hline
\end{tabular}

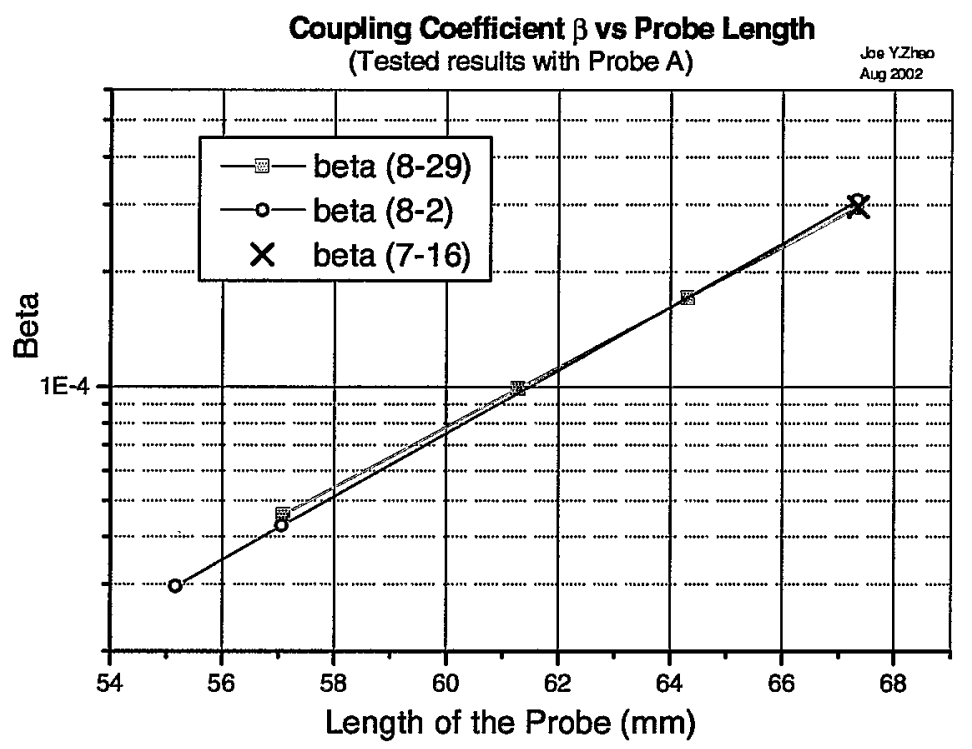

Fig.13. Dependence of coupling coefficient on launcher length 


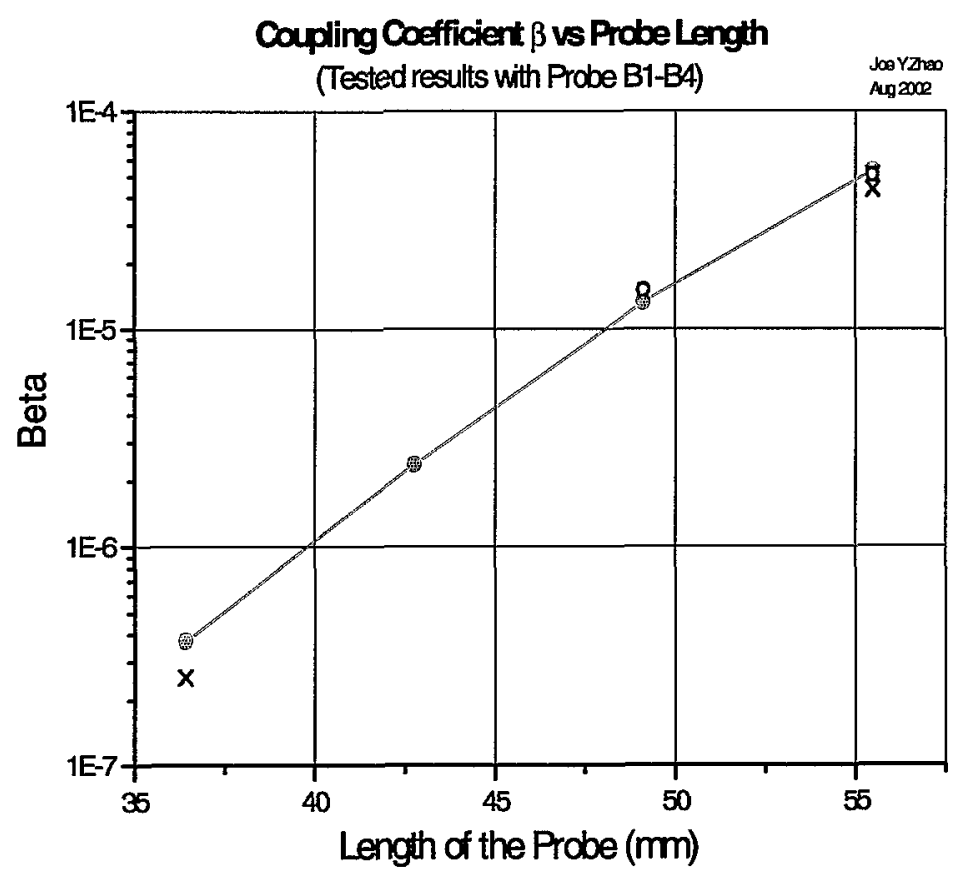

Fig.14. Dependence of coupling coefficient on pick-up length

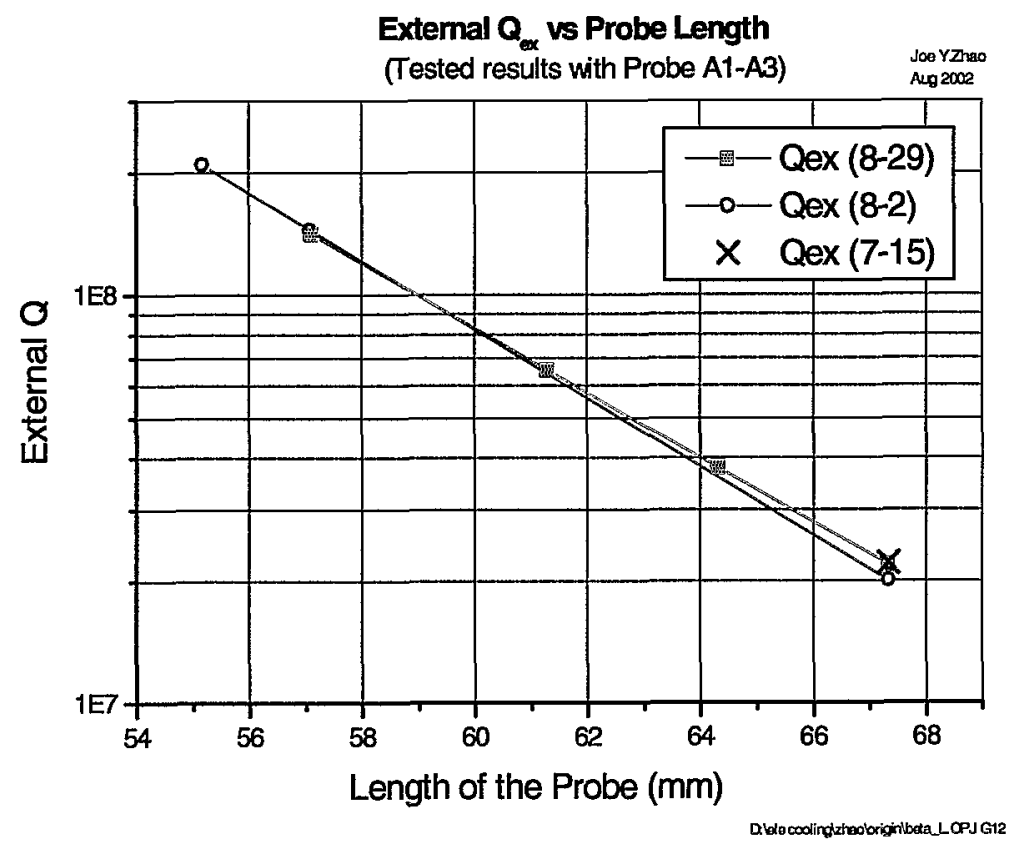

Fig.15. Dependence of external $Q$ on launcher length 


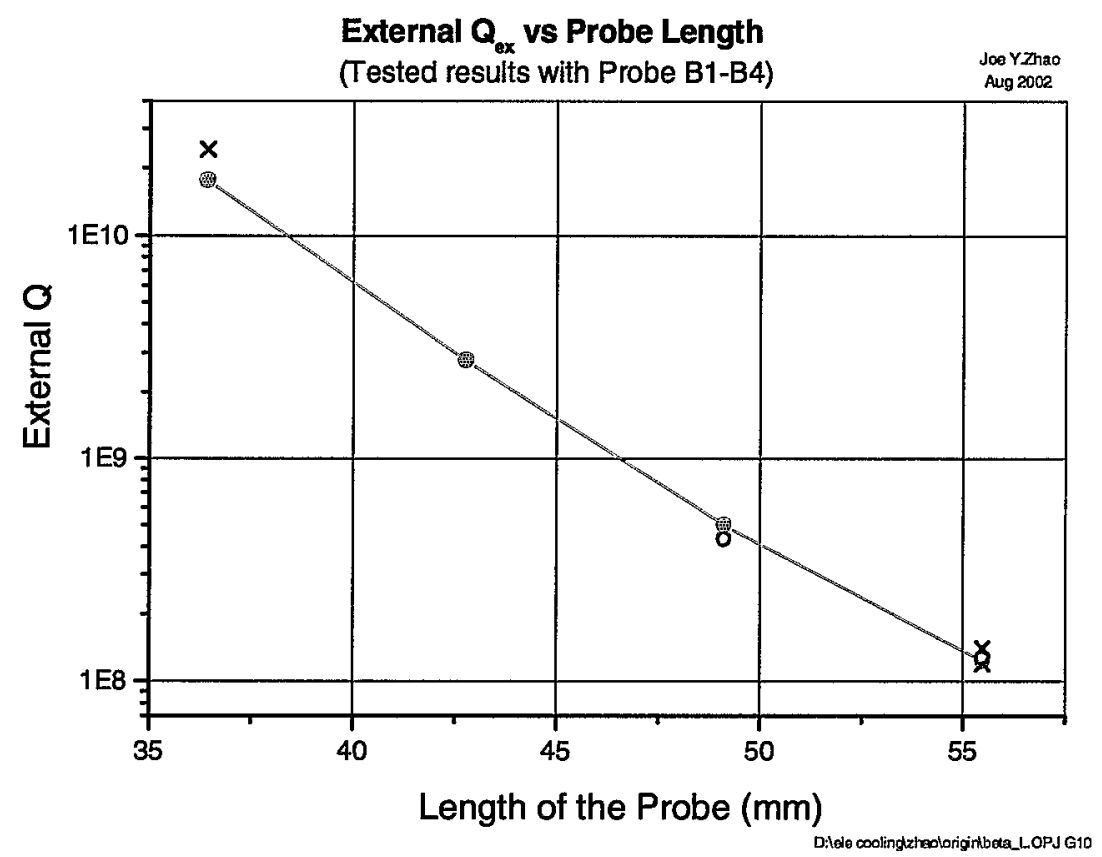

Fig.16. Dependence of external $Q$ on pick-up length

The coupling coefficient and external $Q$ are the most important parameters that need to be determined. From the description above, one can correct the resonant frequency from (23) and the Q value from (41) and (42).

The curves show good results within a reasonable error and thus justify the method in this note.

\section{ACKNOWLEDGEMENT}

This work is supported by the project of "Electron Cooling" headed by Ilan Ben-Zvi, in cooperation with AES. The cavity experiment is managed by Triveni Srinivasan-Rao and Mike Cole of BNL and AES, respectively. Mike Cole participated the cavity measurements in all aspect.

\section{References}

[1] Mike Cole, private communication

[2] Yongxiang Zhao, "SC cavity cross-talk measurement and analysis", presented in Superconducting Cavity Gun Meeting, Advanced Energy Systems, NY, July 22, 2002.

[3] Yongxiang Zhao, "Test report for SC cavity", Aug. 9, 2002.

[4] Yongxiang Zhao, "The report for the SC cavity measurement", Sept.5, 2002. 